\title{
Design of A Power Quality Monitoring System Based on MSP430
}

\author{
Wang Qiang \\ School of Information Science \& Engineering \\ Lanzhou University \\ Lanzhou, China \\ e-mail: wangq101419@126.com
}

\author{
Pang Zhaolong \\ School of Information Science \& Engineering \\ Lanzhou University \\ Lanzhou, China \\ e-mail: pzlzhaolong@126.com
}

\author{
Ma Jianlin \\ School of Information Science \& Engineering \\ Lanzhou University \\ Lanzhou, China \\ e-mail: majianlin2010@gmail.com \\ Liu Yingjie \\ School of Information Science \& Engineering \\ Lanzhou University \\ Lanzhou, China \\ e-mail: ljliu@lzu.edu.cn
}

\begin{abstract}
A power quality monitoring system based on MSP430 is proposed in this paper. The monitoring system uses the high accuracy electronic energy measurement integrated circuit ATT7022E and low power consumption microcontroller. The monitoring system can monitor voltage, current, active power, reactive power, apparent power, active energy, reactive energy, power factor, phase difference, and frequency. The communication function realized the data transmission by General Packet Radio Service. It is showed that the monitoring system is not only an accurate mete of electrical energy, but also to accurately detect the abnormality and record it. The monitoring system is convenient to the power system.
\end{abstract}

Keywords-Power Quality; MSP430; ATT7022E;

\section{INTRODUCTION}

Along with the development of the computer science, customers require high level power quality than ever before, so power quality has become an important concern to customers as well as utilities and facilities [1][2]. The requirement of a portable system which can monitor electrical parameters, detect and record abnormalities in real time is urgent. There have been major efforts made in development of power quality monitoring system. In [3][4], two power quality monitoring system which can detect fault and power fluctuations in real time were proposed. Zhang et al. [5] developed an Internet-based power quality monitoring system for remote power quality inspecting and analyzing. In [6], a monitoring and analysis system using LabVIEW is developed. Marta et al. [7] proposed a power quality monitoring system to be implemented on an FPGA. However, there are many drawbacks like poor accuracy and lack of configurability. this paper contra posed the above points and the applied value of project, it designed the power quality monitoring system based on MSP430.It is a cost effective, reliable and high accurate system which can be used in a single phase system or a three phase system.

In this paper, a power quality monitoring system based on MSP430 is designed. The paper is broken down into the following sections: after this introduction Section 2 describes the hardware design; Section 3 then describes the software design; the last section draws the final conclusions from the work carried out.

\section{HARDWARE DESIGN}

\section{A. Hardware Architecture}

In the proposed system, the hardware consists of potential transformer (PT), current transformer (CT), ATT7022E, MSP430F2619, Keypad, LCD, EEPROM, Real Time Clock and GPRS module. The simplified system architecture is show in figure 1.

\section{B. The Descriptions and Features about ATT7022E}

ATT7022E is a high accuracy 3-phase electronic energy metering chip which is suitable for 3-phase 3-wire and 3phase 4-wire. It incorporates 7 second-order sigma-delta ADCs, reference circuitry and the entire signal processing required calculating power, energy, RMS data, power factor and frequency. ATT7022E is suitable for measuring active power, reactive power, apparent power, active energy, and reactive energy for each phase and 3 phases combined; it is also suitable for measuring voltage RMS, current RMS, power factor, phase difference, and frequency [8]. ATT7022E supports software calibration for gain, phase. ATT7022E provides a SPI interface, SPI interface is used to transfer data to and from host MCU for all measuring result and calibration data.

\section{Analog Input Circuits}

Analog input circuits are shown in figure 2 and figure 3. Fig.2 is voltage sampling input circuit and Fig.3 is current sampling input circuit. The sampling voltage input Metering chip through transformer. On the one hand, the transformer isolates metering chip from grid, thus can obtain good 


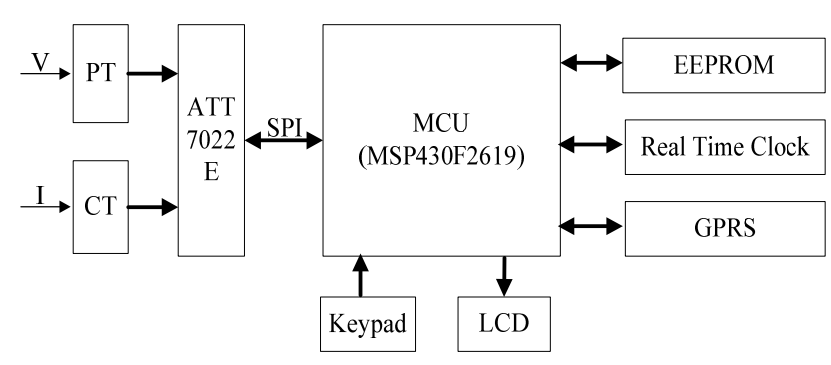

Figure 1. Simplified system architecture.

anti-jamming performance; on the other hand, PT and CT convert large voltage and current signals to low voltage and current signals. After adjustment and filtering, the low voltage and current signals finally input ATT7022E. ATT7022E incorporates 7 second-order sigma-delta ADCs, $\mathrm{V} 1 \mathrm{P} / \mathrm{VIN} 、 \mathrm{~V} 3 \mathrm{P} / \mathrm{V} 3 \mathrm{~N}$ and $\mathrm{V} 5 \mathrm{P} / \mathrm{V} 5 \mathrm{~N}$ as current input channel, V2P/V2N 、 V4P/V4N and V6P/V6N as voltage input channel, V0P/V0N as zero sequence current input channel. Less than $0.1 \%$ nonlinear error when current RMS in range of $0.1 \mathrm{mV}$ to $500 \mathrm{mV}$; less than $0.1 \%$ nonlinear error when voltage RMS in range of $0.2 \mathrm{mV}$ to $500 \mathrm{mV}$.

\section{The Interface Circuit between ATT7022E and MSP430}

ATT7022E has an internal SPI serial communication interface, which use passive working mode. The SPI have 2 controlled lines and 2 data lines: CS, SCLK, DIN, and DOUT. SPI communication interface is connected to MSP430F2619 as sketch map show in figure 4. Every SPI signal line connects a small resistance to prevent possible disturbance. This resistance associates with a capacitance in chip's input port can compose a low pass filter, which could eliminate surge in SPI interface.

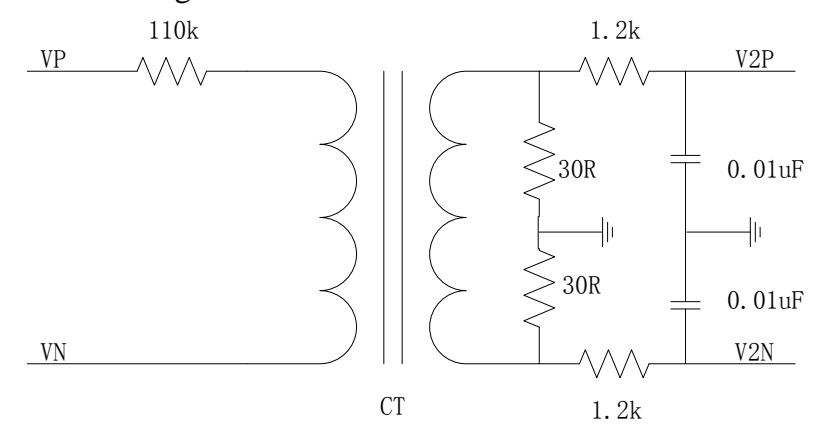

Figure 2. Voltage sampling input circuit.

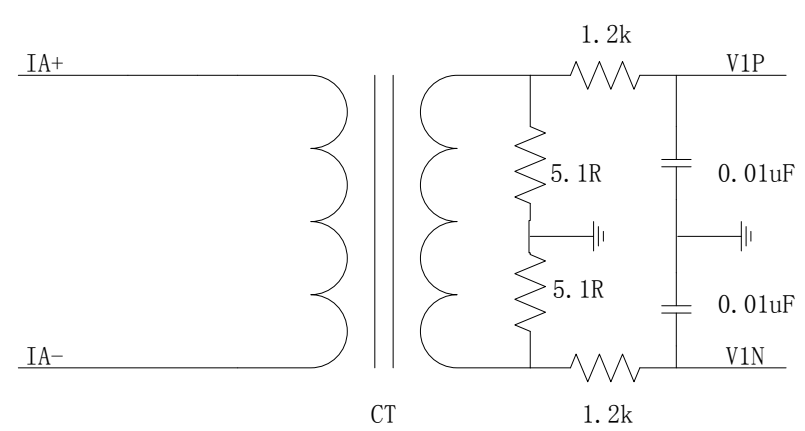

Figure 3. Current sampling input circuit.

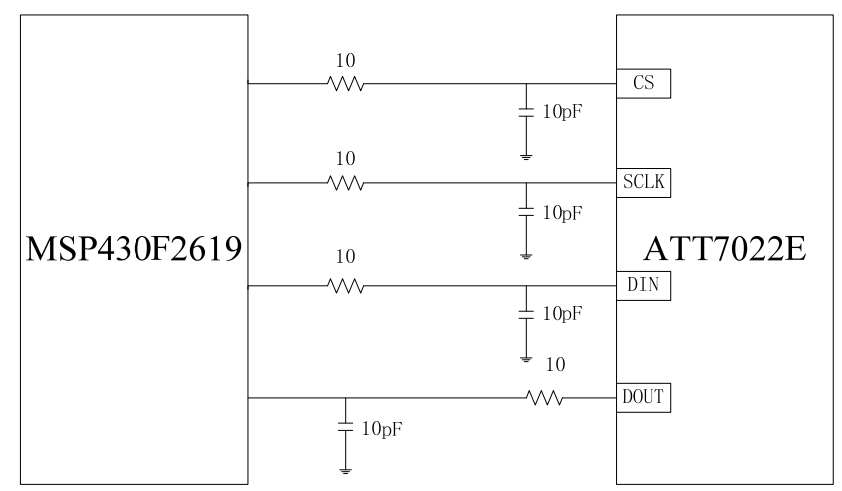

Figure 4. The interface circuit between ATT7022E and MSP430F2619.

\section{SOFTWARE DESIGN}

\section{A. Software Architecture}

The software development environment of the proposed system is across compiler IAR Embedded Workbench which is designed for MSP430 micro-controller by IAR Company. The design of system software can be divided into initialization, ATT7022E calibration, reading data from ATT7022E, key event processing, show information on LCD, store data in EEPROM, communication, and so on. The flow chart of the system software is shown in figure 5 . 


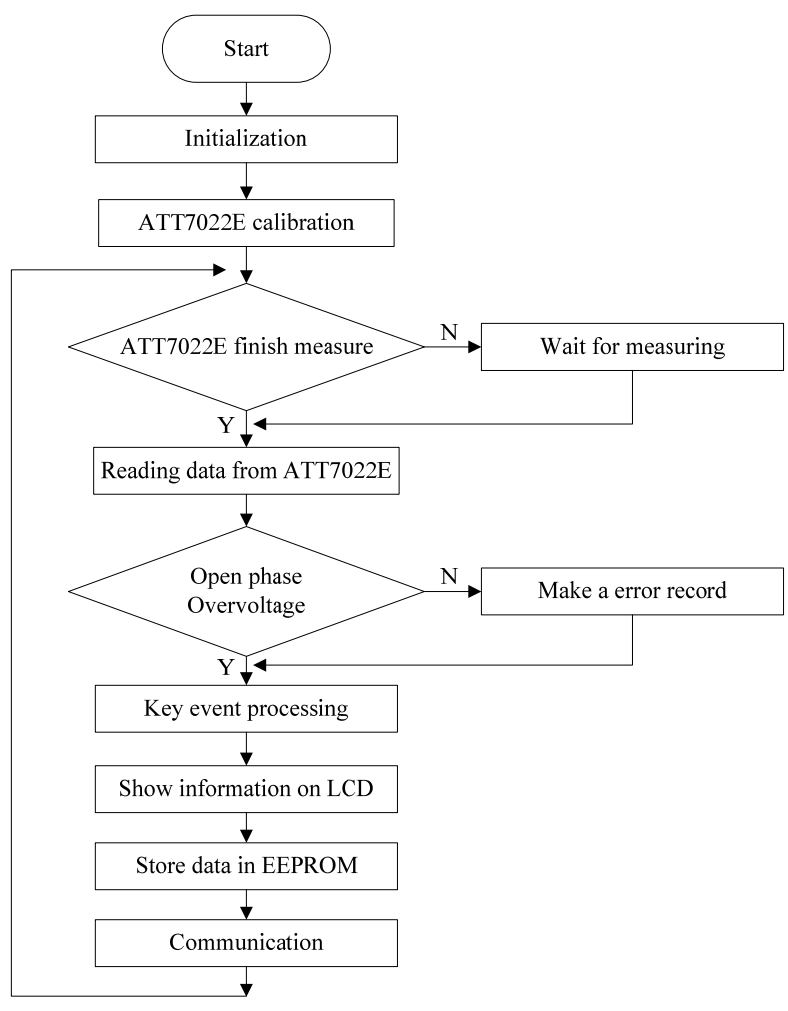

Figure 5. Flow chart of system software.

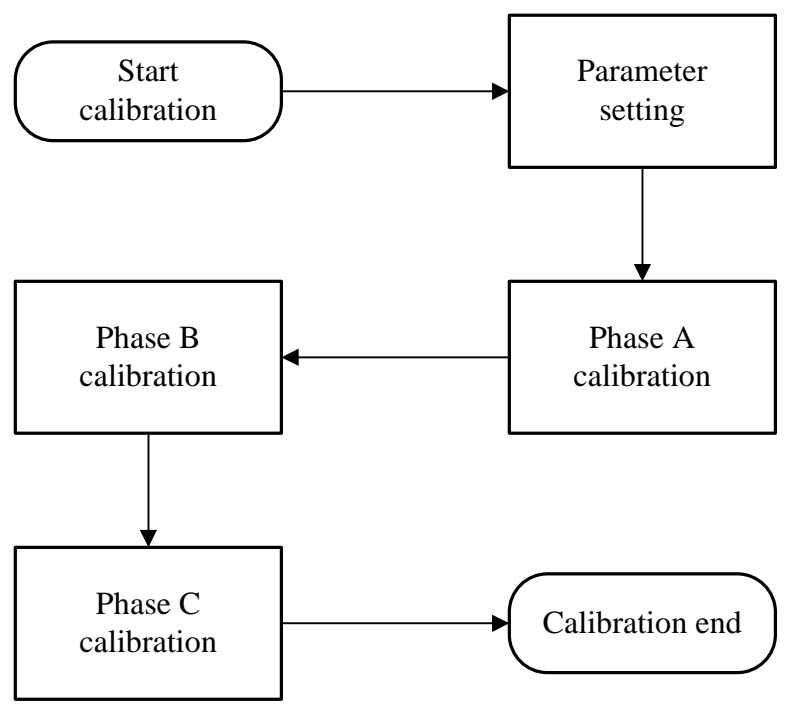

Figure 6. Flow chart of calibration.

\section{B. Calibration}

The standard energy meter is necessary when calibrate energy meter which designed using ATT7022E. CF1 should be connected to the standard meter, then calibrate according to error reading in standard meter. ATT7022E need only calibrate active power; the reactive power need not be calibrated. The flow chart of calibration is shown in figure 6 .

\section{CONCLUSION}

In this paper, a power quality monitoring system is a method which using an energy measurement IC ATT7022E and MSP430 microcontroller applied to the power system fields. The proposed system has the characteristics of steadiness, reliability, fast data acquisition and inexpensiveness. All these advantages have made it the ideal system for remote power quality monitoring.

\section{REFERENCES}

[1] Dong-Jun Won; Il-Yop Chung; Joong-Moon Kim; Seung-Il Moon; Jang-Cheol Seo; Jong-Woong Choe. "Development of Power Quality Monitoring System with Central Processing Scheme" Power Engineering Society Summer Meeting, IEEE Press, July. 2002, pp. 915-919, doi: 10.1109/PESS.2002.1043371.

[2] Meyer, Jan; Kilter, Jako; Howe, Bill; Zavoda, Francisc; Tenti, Liliana; Gordon, Jose Maria Romero; Milanovic, Jovica V. "Contemporary and Future Aspects of Cost Effective Power Quality Monitoring Position Paper of CIGRE WG C4.112” Electric Power Quality and Supply Reliability Conference (PQ), June. 2012, pp. 1-6, doi: 10.1109/PQ.2012.6256208.

[3] Yingkayun, K.; Premrudeepreechacharn, S.; Oranpiroj, K. “A Power Quality Monitoring System for Real-Time Fault Detection” Industrial Electronics, July. 2009, pp. 1846-1861, doi: 10.1109/ISIE.2009.5213582.

[4] Yingkayun, K.; Premrudeepreechacharn, S. "A Power Quality Monitoring System for Real-Time Detection of Power Fluctuations" Power Symposium, Sept. 2008, pp. 1-5, doi: 10.1109/NAPS.2008.5307405.

[5] Ming Zhang; Kaicheng Li. “A Power Quality Monitoring System over the Internet” Information Science and Engineering (ICISE), Dec. 2009, pp. 1577-1580, doi: 10.1109/ICISE.2009.136.

[6] Li Penghui; Zhao Lijie; Bai Haijun; Zhang Yanhua. "Power Quality Monitoring of Power System Based on Spectrum Analysis" EProduct E-Service and E-Entertainment (ICEEE), Nov. 2010, pp. 1-4, doi: 10.1109/ICEEE.2010.5661359.

[7] Ruiz-Llata, M.; Guarnizo, G.; Boya, C. "Embedded Power Quality Monitoring System based on Independent Component Analysis and SVMs” Neural Networks (IJCNN), Aug. 2011, pp. 2229-2234, doi: 10.1109/IJCNN.2011.6033506.

[8] HiTrendtech: ATT7022E User manual, http://www.hitrendtech.com/download/ 